Article

\title{
Calligraphic Glass: Making Marks with Glass
}

\author{
Ayako Tani
}

North Bridge DTP, University of Sunderland, Sunderland SR1 3SD, UK; ayako.tani@sunderland.ac.uk

Received: 30 November 2018; Accepted: 3 January 2019; Published: 15 January 2019

\begin{abstract}
Calligraphic glass is one of the newer developments in contemporary glass art. The long-standing tradition of calligraphy is a rich source of inspiration for the glass artist, glass being a relatively new material for spontaneous mark-making. The investigation of the calligraphic quality of artworks ultimately leads to a discussion of human body movement, because mark-making is fundamentally a kinetic exercise. Often, the artist's psychological and physical state is more important than the resultant marks. Building upon my doctoral research, which introduced the basic idea and techniques of 'calligraphic lampworking', this paper develops the discussion mainly regarding how the artist's body movement may be manifested as spatial traces made from glass lines.
\end{abstract}

Keywords: glass; lampwork; calligraphy; line; movement

\section{Introduction}

The interdisciplinary research field of calligraphy and glass has been thriving in recent years. A number of practice-based researchers specialising in a variety of glass techniques, namely furnace-work and cold-work (Wang 2012), lampwork (Tani 2014), inside painting (Guo 2016), pâte de verre (Qu 2017) and kiln casting (Labatt 2018), have discussed the material characteristics of glass in comparison to ink and developed the uses of glass for calligraphic expression. Current doctoral projects in the UK include a development of calligraphic glass sculpture using metal oxides for tones (Shi 2018) and the recrafting of waste glass (Pailing 2018), both at the University of Sunderland. ${ }^{1}$ Pailing's research does not directly refer to calligraphy, although she is interested in the calligraphic quality found in waste pieces of glass.

Calligraphy has been a medium of self-expression for centuries. This is in contrast to glass, which was only freed from the factory environment for artists through the Studio Glass Movement in the latter half of the twentieth century. Glass is a relatively new material for artists, especially for those who embrace the spontaneous flow of molten glass and accidental shapes over the perfect control of the material. Perhaps it is natural for these glass artists to seek inspiration for mark-making from the long-standing tradition of calligraphy.

I research and practise the lampwork technique, where glass rods and tubes are melted and shaped on an oxygen-propane burner. ${ }^{2}$ My doctoral research applied anthropologist Tim Ingold's (2007, pp. 39-52) 'taxonomy of lines' to the analysis of various line forms in glass art, developing additional categories of lines from the point of view of the lampwork practitioner. Most importantly, I advocated the use of the term 'spatial traces' (Tani 2014, pp. 91-94) to describe how molten glass can record its own trajectory when drawn by a movement in space. I argued that

1 University of Sunderland accommodates the International Research Centre for Calligraphy (IRCC) and the National Glass Centre (NGC), creating a thriving environment for calligraphic glass research.

2 The name 'lampwork' is derived from the use of oil lamps, which were employed for glassmaking in the 17th century when this technique developed in Europe. Today, burners for glasswork often use propane and oxygen gas sourced from liquid gas tanks. 
glass is a rare material which enables the embodiment of spatial traces, and I developed the concept of 'calligraphic lampworking' to demonstrate the idea of capturing the hand's movement in glass (Tani 2014, pp. 215-46).

Over the last several years, I have demonstrated and lectured widely in the UK and China. These encounters through teaching were beneficial not only for the dissemination of research but also for observing people's reactions to the idea of using glass as a drawing medium. A series of interviews with calligraphers who were undertaking projects at the International Research Centre for Calligraphy at the University of Sunderland raised interesting suggestions about the criteria of calligraphic marks. The hand and body are the transmitting devices from brain to the material, but often in calligraphy, the body movement itself becomes the centre of philosophical attention as it is said that 'calligraphy is in essence an art of movement' (Billeter 1990, p. 11). For a further development of calligraphic glass, this paper addresses the artist's body movement and how it might appear and be recorded in glass shapes.

\section{Mark-Making with Glass}

\subsection{Capturing Spatial Traces}

Ingold (2007) introduced a taxonomy of lines based on his thorough observation of various lines and line-making activities. He identified the categories of 'threads', 'traces', 'cut, crack and crease', 'ghostly lines' (pp. 41-50), and the additional motley category of the 'lines that don't fit', which accommodates exceptions (p. 51). He also noted that the majority of lines in the world would fall into the categories of either threads or traces. Threads are lines that can suspend themselves in three-dimensional space, whereas traces need to be supported by a surface. Threads are a volumetric entity, and traces are areal. In many cases, it is also observed that threads are more static and traces are kinetic. This is because traces are often the track marks of something that moved across the surface, as is the typical case for brushstrokes drawn on paper. Threads could be kinetically drawn as well if ink could be painted onto air, which is normally inconceivable. Therefore, generally, drawing has a two-dimensional basis.

Based on this argument, I added 'spatial traces' (Tani 2014, pp. 91-94) as a contrast to the usual traces trapped on a surface. A good example of a spatial trace is the pattern drawn by a musical conductor with their baton. Although it is intangible, it is not a hypothetical construct but a real mark that can convey meaning. Tavs Jorgensen's glass bowl (Figure 1) is an example of the embodiment of a spatial trace in glass. Jorgensen employed a drawing device connected to the computer, and he translated his hand's movement of drawing a circle into a crown-shaped metal mould. The contour of the mould was made to the shape of the trajectory of his hand. A sheet of glass was slumped over this mould and created into a round bowl with the rim reconstructing the trajectory. The spatial trace was firstly only captured by the eyes of computer, and it was then visualised in glass.

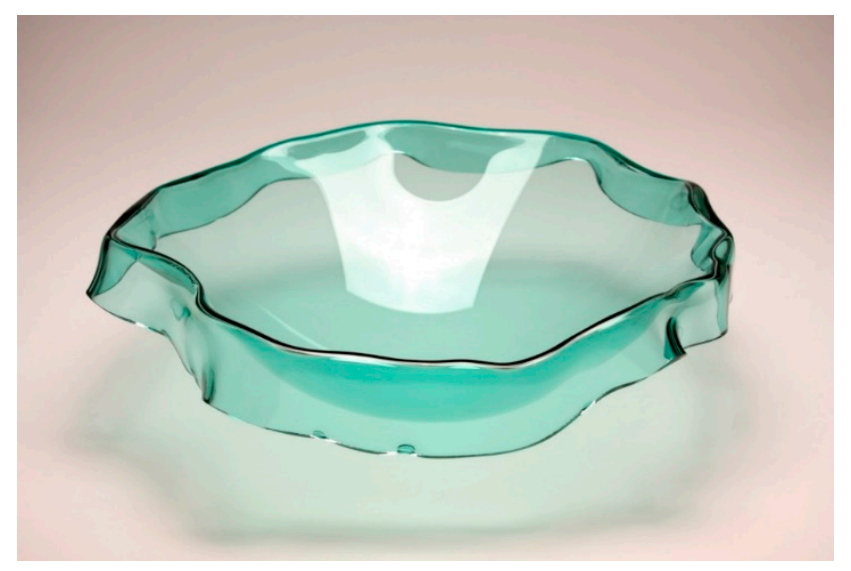

Figure 1. Large Green One-liner Bowl (2008) by Tavs Jorgensen. 


\subsection{Calligraphic Lampworking}

Lampwork glass can give spatial traces tangible forms, because molten glass flows freely and it quickly cools and sets into a shape. The bodily action of stretching molten glass in the air is similar to that of drawing in ink with a brush. Just as the ink mark is a trace of the movement of the hand and brush, the glass, while molten, forms a spatial trace embodying the movement of hot glass manipulated by hand. The line of glass, after cooling, can suspend itself in space and be independent from a surface, which meets the criteria of 'threads'. However, spatial traces remain as a separate category, as they are characterised by their formative process and are a record of spatial movement.

The spatial traces embodied with glass are calligraphic. Spatially stretching glass and dragging brush on paper are both kinetic operations which create incremental, spontaneous, and irreversible lines. Among various different glass-forming techniques, lampworking is close to ink calligraphy, because the artist can move the glass rod with one hand in the similar way to handling a brush.

Ghost (Figure 2) confirms the technical feasibility of using molten glass in a calligraphic manner, and it demonstrates the amalgamation of calligraphy and the visual connotation carried by clear glass. In this work, four Japanese characters “ゆうれい” (pronounced as yu-u-re-i) meaning 'ghost' are written in clear glass. These four characters are graphically arranged in the ghostly figure inside the frame, and the frame is suspended from the ceiling to achieve the floating effect. The fleeting shadow also forms a ghostly trace. As the suspended sculpture slowly sways, the projection of the shadow comes in and out of focus, enlarging and blurring.

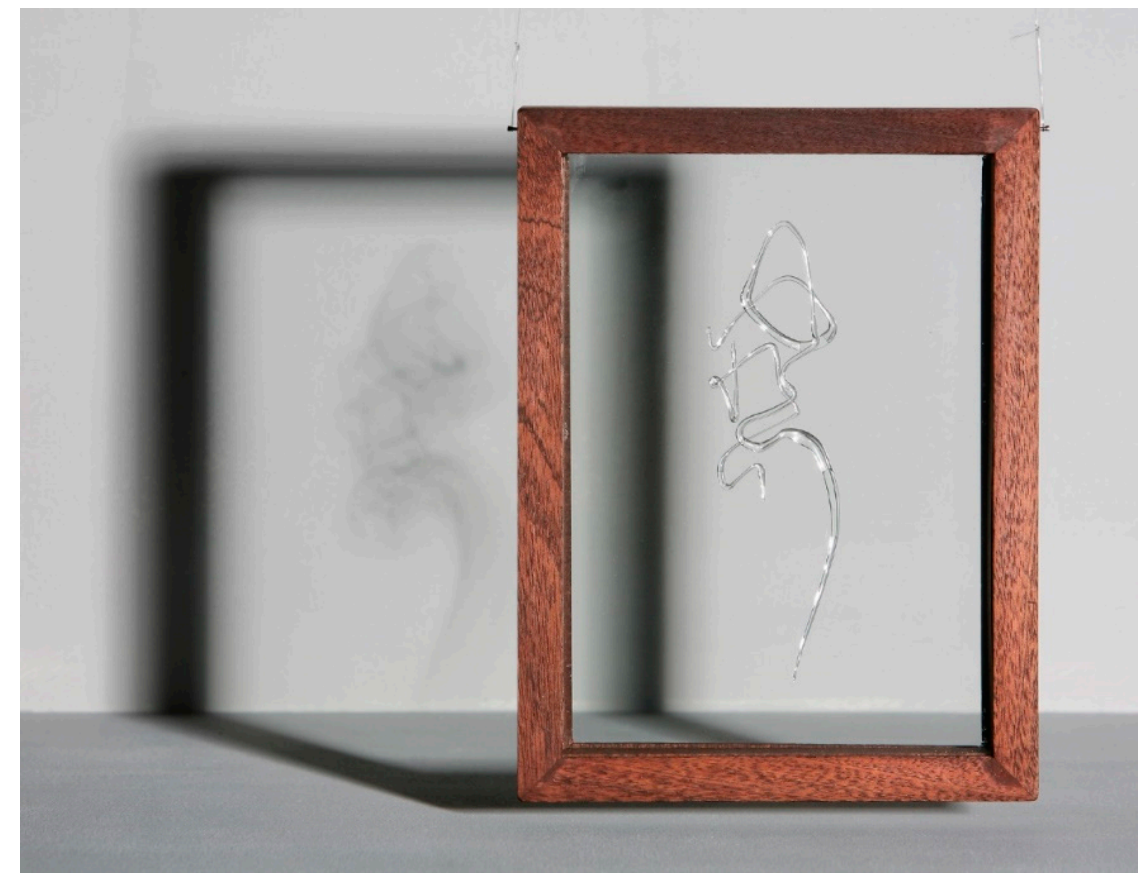

Figure 2. Ghost (2010) by Ayako Tani. Lampworked borosilicate glass and wood frame. Variable size.

This work questions our perception of dimensionality. It is a mass of material, which is physically a three-dimensional object, but people see and discuss the dimensions in artwork more conceptually and intuitively. It can be argued that Ghost is more than a three-dimensional object because the shadow adds extra layers of meaning to the work. On the other hand, it could be seen as less than three-dimensional because the clarity of glass flattens the volume. Being able to observe the full shape from a single viewpoint normally only applies to two-dimensional work such as painting. Sculptures made of opaque material must be viewed from behind to appreciate their entire shape. It is a special condition of clear material like glass that the back side of the object can be seen through the volume. In addition, the volume of glass can enable the viewer to see optical effects caused by 
reflection and refraction. The dimensionality of clear glass is difficult to quantify and, therefore, I call it 'multi-dimensional' or 'trans-dimensional' (Tani 2014, p. 109).

\subsection{Focus and Sub-Consciousness}

Calligraphers say that brush strokes can convey something about the core of an individual's inner quality. It is because the artist's state of mind affects their actions, and appears in the resultant line. Therefore, the investigation and development of the calligraphic line ultimately leads to a philosophical discussion about the relationship between the mind and bodily movement. A notable theme is the question of whether more successful work comes from a focused mind or is the result of the subconscious.

The glass marks shown in Figure 3 are waste glass pieces which artist Helen Pailing collected from the studio of Brian Jones, a scientific lampworker at the National Glass Centre. As seen in the picture, she thoroughly painted them in florescent orange paint to highlight their shapes. Each of these pieces was originally an excess section of a glass tube, which was heated, squashed, twisted, and pulled away in the course of making some kind of product. These marks were produced by Jones's hands and they freeze the moment of when they were made. However, Jones's attention must have been paid to the product he was making at that time, and probably he removed and threw these fragments into the bin as part of a subconscious routine.

Rejections are slightly different from waste fragments. Figure 4 shows rejections of my artwork Ghost (Figure 2). I drew a hundred 'ghosts' to get one piece which satisfied my aesthetic criteria, and all other weaker pieces were rejected. Each of these pieces was drawn with a focused mind.

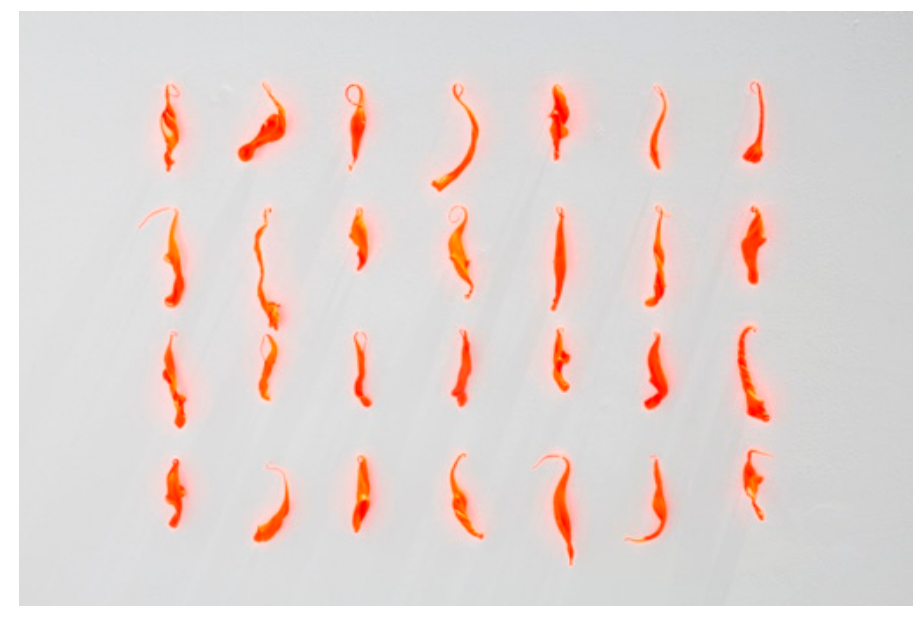

Figure 3. Vibrant Matter (2017) by Helen Pailing. Waste glass and acrylic paint. Variable size.

Discussions with calligraphers reveal that focus and subconsciousness, which are seemingly oppositional states of mind, might be closely linked. Subconsciousness is perhaps shrouded in consciousness. One of the calligraphers used a tennis player as an example to explain this. When the top players hit the ball, they do not think about their steps or raising their arm but their body automatically reacts. They are extremely focused, not to take control of their body movement, but to let it happen without the interposition of their mind. And, this state can only be achieved by considerable practice and experience. My repetitive ghost-making was a focused process of practice and experience development in order to polish my mental and technical skills so that my movements almost became subconscious. Pailing's collection of waste marks was an alternative way to achieve the happy accident of a master maker. In this case, subconsciousness was contrived by blocking the communication between the hand and the mind by using two individuals. 


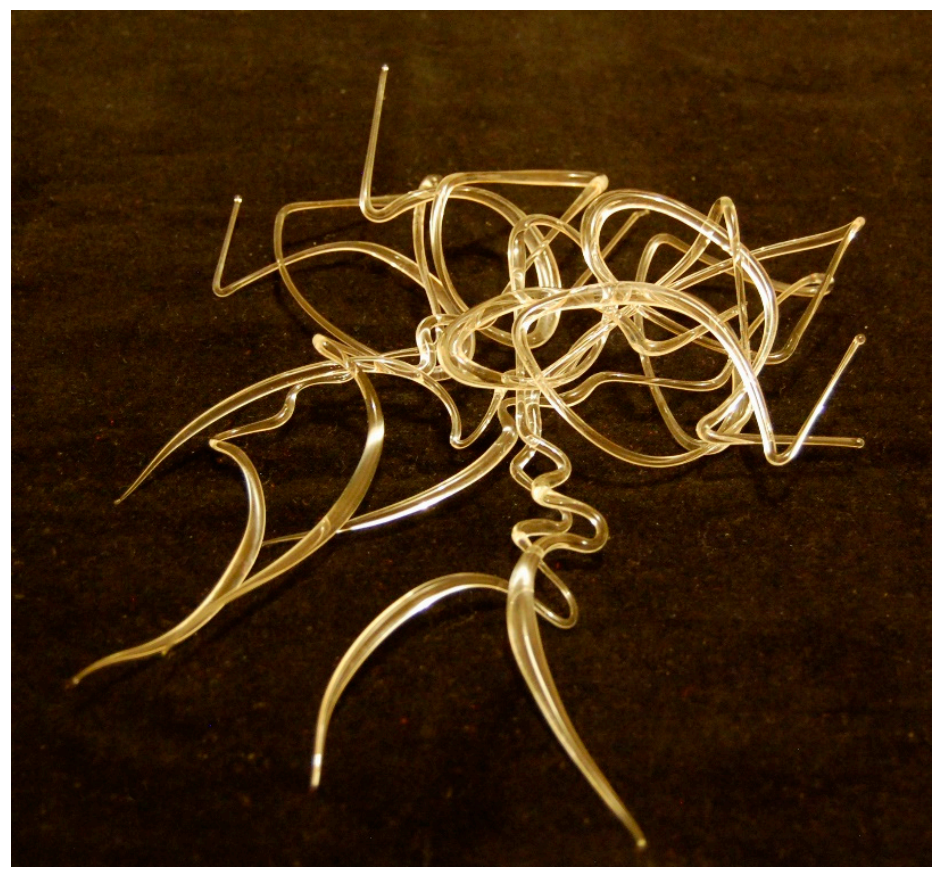

Figure 4. Rejected parts of Ghost by Ayako Tani.

Getting focused to surrender the mind to subconsciousness is the practice of Zen meditation. One of the exercises is called Ensō drawing, where a full circle is drawn with one uninhibited brushstroke. Zen monks have drawn these over the ages for "expressions of their understanding of life" (Flint-Sato 2014, p. 39). Sunny Wang brought this practice to glass (Figure 5). She took molten glass from the furnace and drew a circle within one breath. She created an installation of a hundred of these glass circles, each representing one of her breaths. Controlling breath is an integral part of calligraphic mark-making. The 'Chi' — the flow of energy-in East Asian philosophy literally means breath. Calligrapher Manny Ling (2008, p. 174) suggested that the 'Chi' and the sense of putting vital energy into mark-making can be shared by different cultures.

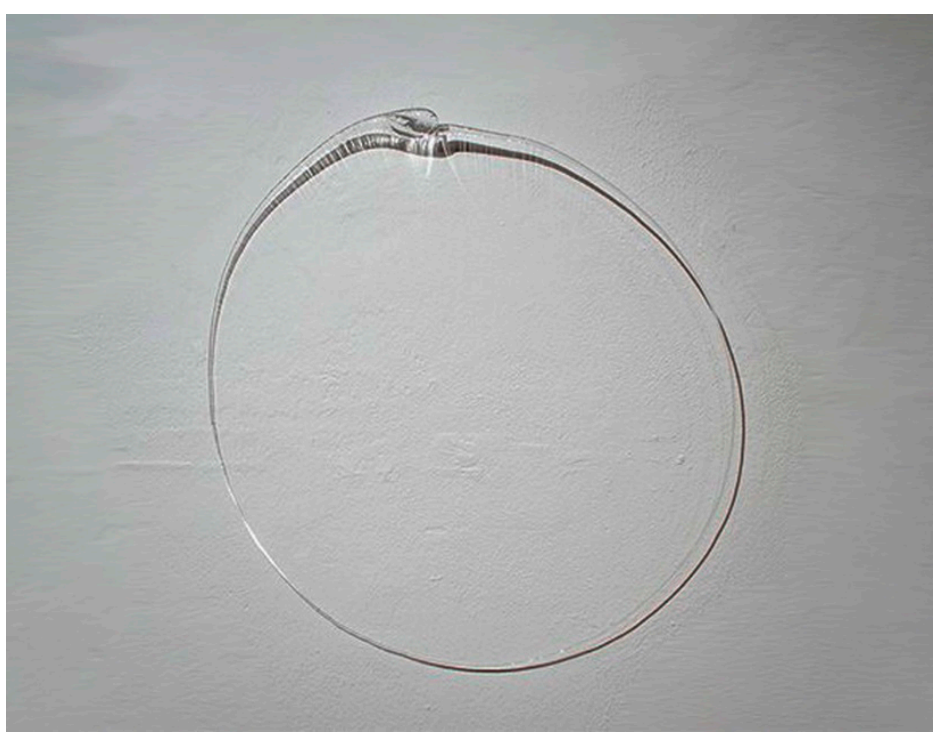

Figure 5. The Glass Circles (2013) by Sunny Wang. Detail. 


\subsection{Marks of Life in Glass}

We strive to leave our mark on the world, but sometimes this is achieved unknowingly. Figure 6 shows a section of a lease-rod reclaimed from a vintage weaving machine. The machine had a set of these rods, which kept warp yarns in position. Glass was used for this purpose because it did not damage the strings like metal and lasted longer than wooden rods. On the surface of the rod, a set of four notches can be observed at equal spaces. These are 'reductive traces' (Ingold 2007) abraded by the miles of threads which travelled over the rods over time. These marks were invisible when the actual 'threads' were running over them, and these unsubstantial 'ghostly lines' (Ingold 2007) only became visible after the machine was dismantled. The marks invite us to imagine the lost threads. The centre of attention of this object is not the marks left, but the threads that made them.

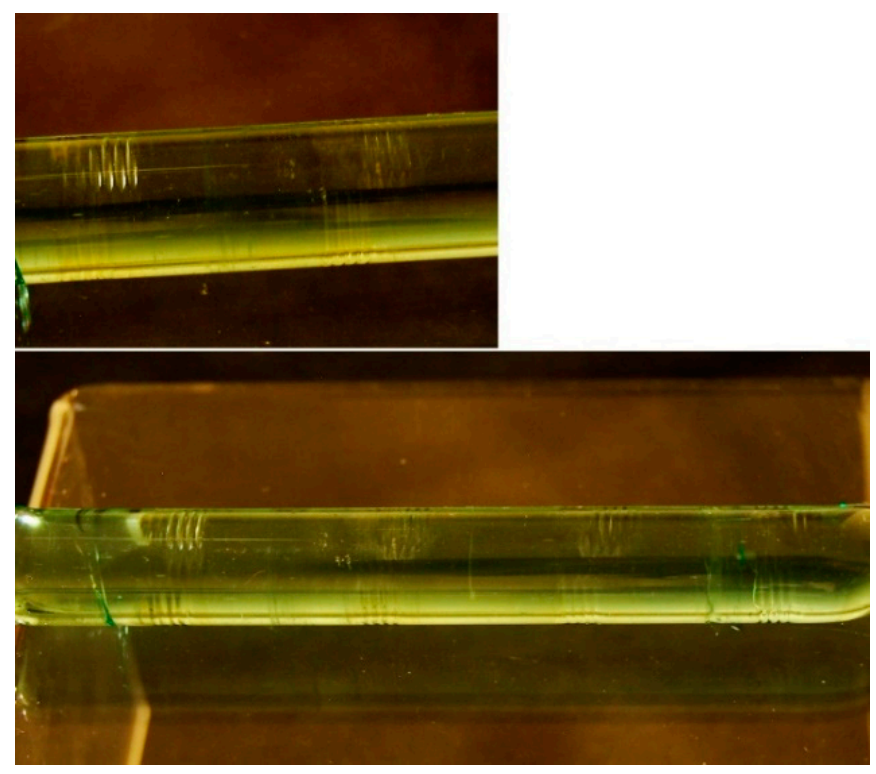

Figure 6. A section of a lease-rod (found in 2018).

The stress crack is an idiosyncratic mark in glass which is caused by uneven heating and only arises later. When glass is heated partially, stress is caused between the hot and cold areas. It breaks when the stress reaches the limit, sometimes days later, triggered by any slight physical or thermal shock. Glass products are normally annealed to prevent this from happening. Figure 7 shows part of a glass ship, left by the late Peter Coyne, a scientific glassblower who was influential in making novelty glass products in northeast England. The end of the glass rod was nipped and slightly pulled over to create the impression of a ship's bow and keel. The cross-sectional line is the stress crack caused by this heat. Normally, lampworkers give this stress zone a 'lamp-annealing' to prevent such cracks. Lamp-annealing is one of the fundamental techniques of lampworking, where the stress zone is gently heated with a lower temperature flame (i.e., using less oxygen) to release the stress. Since lampworking often includes the partial heating and fusing of small components, this quick annealing method is frequently employed in the course of fabricating a sculpture. This remnant suggests that Coyne was interrupted by a phone call or something of that kind and had to put the piece down without first lamp-annealing it. The crack is a poignant record of him having worked on this piece of glass. 


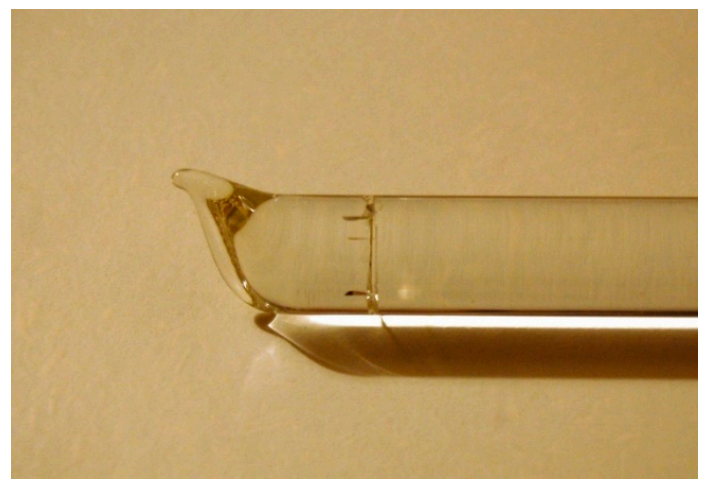

Figure 7. A ship nose with stress cracks (found in 2018).

\section{Studio Practice: Murmuration}

Alongside the theoretical study of mark-making with glass, I have investigated the technical possibility of lampworked glass, seeking a new calligraphic expression. As a result, the Murmuration series was developed. This section introduces the studio practice of this research and explains the developmental path from the initial ideas to the final artwork.

Lampworkers use glass tubes as well as solid rods. Typical products made from tubing are various sorts of containers and blown sculptures such as Christmas baubles. It is also a common practice for scientific lampworkers to fabricate tubes into double- or triple-walled laboratory apparatus. As Ingold (2007) mentions, a tube has a line of air inside and is an exceptional type of line. This dual structure is very visible with clear glass. Building upon this observation, I aimed to develop the use of tubing for calligraphic expression. The practice-led investigation was done through two major artist-in-residence programmes. Firstly, I took an experimental approach for the Emerging Artist in Residence at Pilchuck Glass School in 2015. I made samples of tubes manipulated through various techniques and by different tools, aiming to enumerate all possible shapes obtainable from tubing. Figure 8 shows one of the test pieces made during this period. These are $10 \mathrm{~mm}$ diameter tubes, cut into small sections and fused back together at various angles.

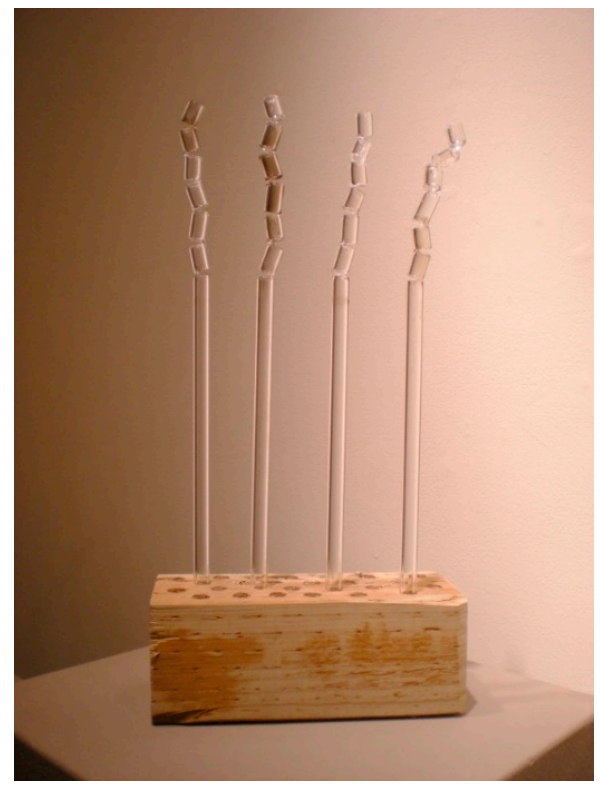

Figure 8. A test of tubing (2015) by Ayako Tani.

Reflecting upon the outcomes of this series of experiments, I concluded that one particular technique to focus on next was that of pushing glass inside the tube and adding embedded textures 
and colours (Figure 9). These are $24 \mathrm{~mm}$ diameter tubes, which have an internal space of $20 \mathrm{~mm}$ for embedding. Each spike was made by heating a small area of the glass using a sharp flame and pushing it towards the middle of the tube with a needle-like metal tool. The size and shape of the spike can be changed by using different tools, and various coloured glass elements can be added. I chose the murmuration of starlings as the inspiration to create new work using this new technique. The dynamic movement that flocks of birds display in the sky seemed to be an appropriate model to learn from and a suitable motif for capturing the flow of energy in glass.

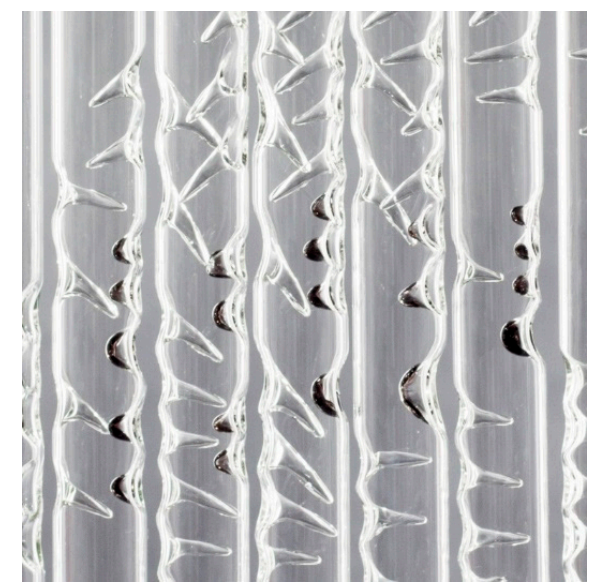

Figure 9. Detail of tubes with embedded textures and colours (2017) by Ayako Tani.

The design and techniques were refined during my residency at JAEA Ceramics Art Centre in China in 2017. Staying in the heartland of calligraphy, I was naturally immersed in the works of masters, saw demonstrations and remembered some traces of knowledge I had learnt at school in Japan. I did oversized sketches (Figure 10) to transfer my whole-body movement into the marks. It was an attempt to experience the sense of getting into the drawing like the Abstract Expressionists had aspired to. At the end of the residency period, this pattern of brush strokes was made into glass using the 'embedded glass technique' explained above. The final piece (Figure 11) was collected by Liling Ceramics Valley Museum in China after the exhibition. Works from this Murmuration series have been selected for several exhibitions, including the British Glass Biennale 2017.

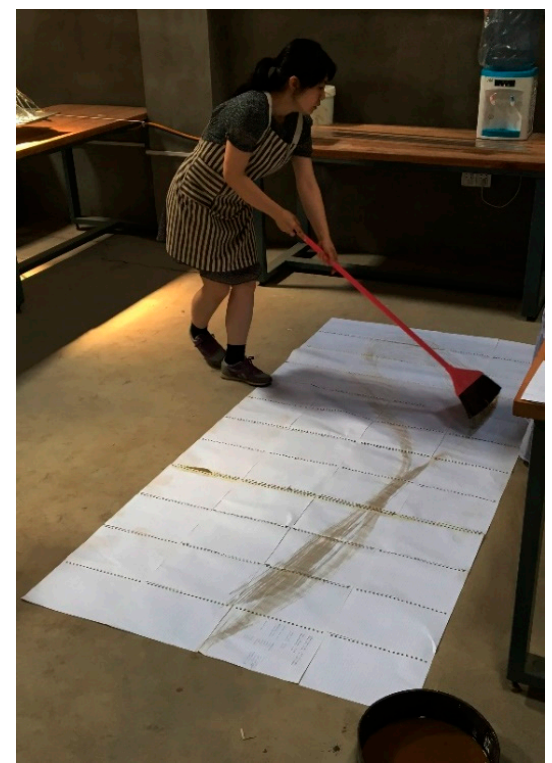

Figure 10. Calligraphic sketch (2017) by Ayako Tani. 


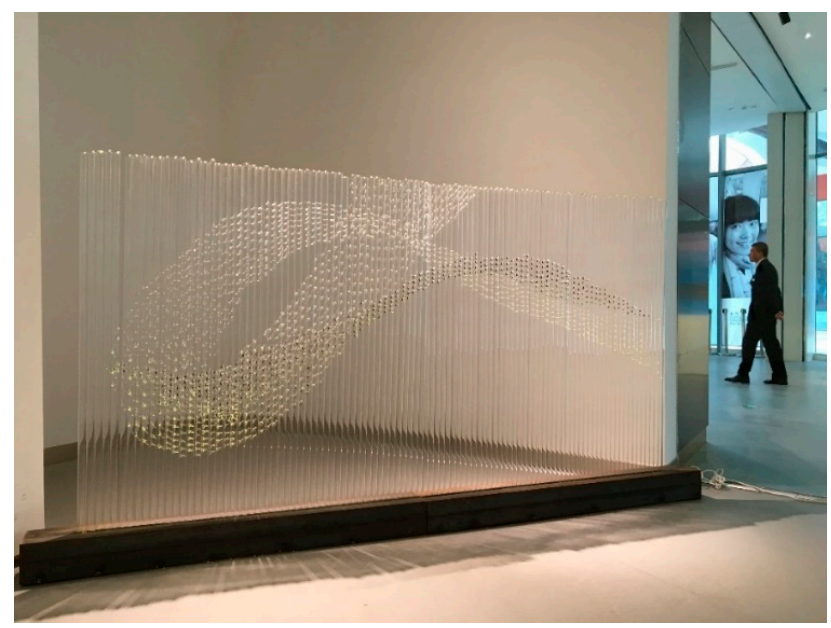

Figure 11. Murmuration no. 3 (2017) by Ayako Tani.

This work translates my mark-making movements on the two-dimensional paper surface into embedded notations in a three-dimensional sculpture. However, this approach differs from that of Ghost, for example, in that massed tubes create the feeling of a canvas or scroll, rather than existing as a free-hanging spatial trace. Although the production of each tube has demanded a considerable level of focus, the whole sculpture attempts to evoke the subconscious, yet virtuoso, movements of a flock of birds in flight or, indeed, a master calligrapher.

Funding: This research received no external funding.

Conflicts of Interest: The author declares no conflict of interest.

\section{References}

Billeter, Jean François. 1990. The Chinese Art of Writing. New York: Rizzoli.

Flint-Sato, Christine. 2014. Sumi Workbook. Osaka: Kaifusha.

Guo, Jianyong. 2016. Inside Painting, as Used for Chinese Snuff Bottles, Suggested as a New Model for Contemporary Glass Art. Ph.D. thesis, University of Sunderland, Sunderland, UK.

Ingold, Tim. 2007. Lines: A Brief History. London: Routledge.

Labatt, Sheila. 2018. Glass as Ink: Seeking Spontaneity from the Casting Process. Ph.D. thesis, Royal College of Art, London, UK.

Ling, Manny. 2008. Calligraphy across Boundaries. Ph.D. thesis, University of Sunderland, Sunderland, UK.

Pailing, Helen. 2018. Recrafting Waste Glass from the National Glass Centre: A Collaboration between Makers and Matter. Northumbria-Sunderland Consortium. Available online: http:/ / northumbria-sunderland-cdt. northumbria.ac.uk/Student-Profiles/Helen-Pailing (accessed on 30 November 2018).

Qu, Jing. 2017. Developing Pâte de Verre Glass Art through the Chinese Philosophy of Wu Wei. Ph.D. thesis, University of Sunderland, Sunderland, UK.

Shi, Dian. 2018. An Investigation into the Application of Metal Oxides in Studio Glass to Form a New Model of Contemporary Glass Calligraphy. Paper presented at Society of Glass Technology Annual Conference, Murray Edwards College Cambridge, Cambridge, UK, September 2-5. Available online: http:/ / cambridge2018.sgt.org/ab-hh-dianshi (accessed on 30 November 2018).

Tani, Ayako. 2014. Multi-dimensional line-drawing with glass through a development of lampworking. Ph.D. thesis, University of Sunderland, Sunderland, UK.

Wang, Sunny. 2012. The Art of Be/Coming through Glass: The Exploration of Studio Glass Art Inspired by Chinese Calligraphy and Framed by Buddhist Practice. Ph.D. thesis, University of South Australia, Adelaide, Australia.

(C) 2019 by the author. Licensee MDPI, Basel, Switzerland. This article is an open access article distributed under the terms and conditions of the Creative Commons Attribution (CC BY) license (http:/ / creativecommons.org/licenses/by/4.0/). 